\title{
On Tensors which are Functions of Vectors
}

N. D. SeN Greta (Tata Institute of Fundamental Research, Colaba, Bombay 5. India)

Abstract. * It has been shown that the most general constant tensor of any rank is the possible algebraic combination of Kronecher tensor $\vartheta_{\Lambda_{1} \mu_{2}}$ and the totally antisymmetric tensor $\Sigma_{\mu_{1}} \ldots \mu_{n}$. Further, the tensor of a given rank $r$, which are functions of vectors $p^{*} s$, are suitable algebraic combinations of the tensor $p_{i_{2}}^{1} p_{i_{2}}^{2} \ldots p_{i_{r}}^{*}$ and the two constant tensors with coefficients which are functions of the scalar products formed by the vectors.

\section{1. - Introduction and formulation of the problem.}

In many physical and geometrical problems, one very frequently comes across tensors which depend only on one or more vectors arising naturally from the given proplem. This motivates one to study the class of tensors which may be formed from one or more vectors. Recently $Z_{I N}$ (1) has tried to study the tensors which depend on the position vector only. The method developed in this paper, which is based on the simultaneons covariance property of the tensor and its argument vectors, is a general one as regards the dimension of the space, the rank of the tensor and the number of independent argument vectors. However, we assume that the vectors are continuously variable and the elements of the tensors are differentiable functions of the components of the vectors which is the case in most of the problems envisaged above.

Let $p^{i} s(i=1,2, \ldots, m)$ be given vectors in $n$-dimensional Euclidean space. The case of pseudo-Euclidean space need not be considered separately as we need not restrict $p_{\mu}^{i,} s(\mu=1,2, \ldots, n)$ to be real; hence, we need not also distinguish between covariant and contravariant tensors. Further, we confine ourselves to those tensors which are associated with the subgroup of the rotation group which is connected to unity. The results may be conveniently extended to that of the full rotation group containing reflections. For obvious reason, the convention of summation over dummy indices is not used and in the text summations are shown explicitly.

(1) G. ZrN, (i) Funeioni tensoriali di un vettore e leggi dellelettronagnetisno, Ann. di Mat. Pura e App. (Volume dedicated to Professor Antonio Srgnorini) 1960 (IV), Vol. L, pp. 341.378 .

(ii) Su una particolare classe di funzioni tensoriali d un tetravetfore e sulla conseguente interpretasione dell'elettromagnetismo di Maxnell-Lorentz, ibid 1969 (IV). Vol. LX1, pp. $30 \overline{0} 1-383$. 
Let $F_{\mu_{1} \mu_{3} \ldots \mu_{r}}$ be a tensor of rank $r$ which is a function of arguments $p^{1}, p^{2}, \ldots, p^{m}$ vectors; the components of the vector $p^{i}$ being $p_{1}^{i}, p_{2}^{i}, \ldots, p_{n}^{i}$. Without any loss of generality we can take $m \leq n$, as there are only $n$ linearly independent vectors. It is needless to mention that one of them may be the position vector having components $x_{\mu}(\mu=1,2, \ldots, n)$. Let us consider an infinitesimal transformation such that the coordinates $x_{\beta}$ are transformed to $x^{\prime}{ }_{\mu}$ given by

$$
\begin{gathered}
x_{\mu}^{\prime}=\sum_{(\theta=1}^{n}\left(\delta_{\mu \mu()}+e_{\mu(\theta)}\right) x_{(\mu)} \\
\sum_{\mu=1}^{n} x_{\mu}^{\prime 2}=\sum_{\mu=1}^{n} x_{\mu}^{2}
\end{gathered}
$$

where $e_{\mu,(\theta)} s$ are infinitesimal parameters of the transformation such that

$$
e_{\mu \leftrightarrow}+e_{(\mapsto) \mu}=0
$$

there are only $n(n-1) / 2$ independent elements. Hence for any vector

$$
p_{\mu}^{\prime i}=\sum_{(\theta=1}^{n}\left(\delta_{\mu, \omega}+e_{\mu(\omega)}\right) p_{\Theta}^{i}
$$

and

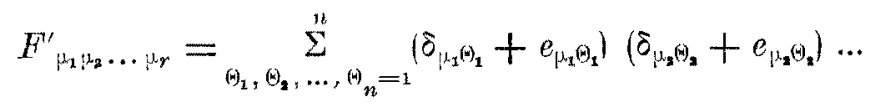

$$
\begin{aligned}
& \ldots \mid E_{\left(1, r \theta_{r}\right.}+e_{\left[1, r\left(\theta_{r}\right)\right.} F_{\Theta_{3}\left(\theta_{3} \ldots \theta_{r} .\right.}
\end{aligned}
$$

which may be written in the simple form, retaining only first order infinitesimal quantities,

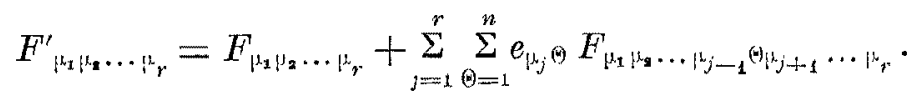

On the other band since $F_{\mu_{1} \mu_{x} \ldots \mu_{r}}$ are functions of $p^{i}$ 's only,

$$
F^{\prime}{ }_{\mu_{1} \mu_{2} \ldots \mu_{r}}\left(p^{i}\right)=F_{\mu_{1} \mu_{2} \ldots \mu_{r}}\left(p^{\prime i}\right)
$$

Substituting for $p^{\prime}$, its expression given by eq. (4) and retaining only first 
order quantities in eq. (7)

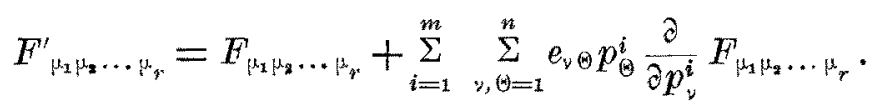

Hence from eqs. (6) and (8),

$$
\begin{aligned}
& \sum_{\substack{\nu,(\theta=1 \\
(\nu>\Theta)}}^{n} e_{\nu \circledast}\left[\sum_{i=1}^{m}\left(p_{\Theta}^{i} \frac{\partial}{\partial p_{\nu}^{i}}-p_{\nu}^{i} \frac{\partial}{\partial p_{\theta}^{i}}\right) F_{\mu_{1} \mu_{1} \ldots \mu_{r}}\right.
\end{aligned}
$$

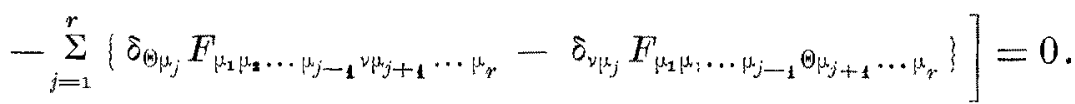

Since $e_{\nu}{ }^{\prime} s(\nu>\theta)$ are independent,

$$
\begin{aligned}
& \sum_{i=1}^{m}\left(p_{\omega}^{i} \frac{\partial}{\partial p_{y}^{i}}-p_{\nu}^{i} \frac{\partial}{\partial p_{\omega}^{i}}\right) F_{\mu_{1} \mu_{2} \ldots \mu_{r}}=
\end{aligned}
$$

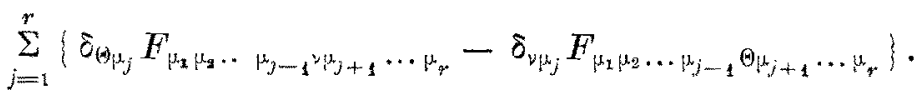

This is the most general equation tensors which are functions of vectors must satisfy. It may be noted that the number of equations is $n^{r+1}(n-1) / 2$ among $n^{r}$ elements of the tensor $F$ of rank $r$, but they are not all independent and the system is compatible. It needs mention here that the set of eq. (10) may be gronped into suitable subsets. One important subsets is the tensors which are totally antisymmetric in their indices. This is nothing but a reflection of the fact that tensors which are totally antisymmetric in their indices form an invariant subspace in the representation of the rotation group.

Finally in the trivial ease of scalars $F_{0}\left(p^{i}\right)$, i.e., tensors of rank zero,

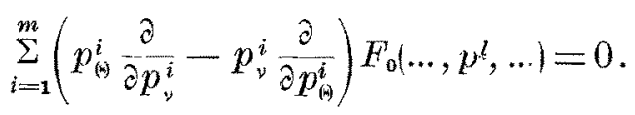

The solutions are

$$
\sum_{\emptyset=1}^{n n} p_{\Theta}^{i} p_{\theta}^{l}, i, l=1,2, \ldots m
$$

(or any function of them); these are simply all possible scalar products of the vectors which are invariant. Thus in the solution of the system 
of eq. (10) arbitrary functions of the scalars $\sum_{(\theta)=1}^{12} p_{(\rightarrow)}^{i} p_{(\rightarrow)}^{l}$, may appear as factors. The number of such functions depend on the rank of the tensor $r$, the number of vectors $m$ and the dimension of space $n$. In the sequel whenever we mention constant coefficients they are all functions of the invariant scalar product.

\section{2. - Constant tensors.}

Before proceding to the solution of eq. (10) in general, let us discuss particular case of tensors which are constants. They satisfy eq. (10) with left hand side zero, hence

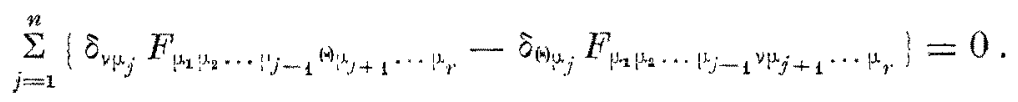

The two well-known solutions of this equation are

(i) the symmetric Kronecker tensor $\delta_{\mu_{1} \mu_{2}}$, of rank 2, and

(ii) the totally antisymmetric tensor $\varepsilon_{\mu_{1} \mu_{2} \ldots \mu_{n}}$ of rank $n$ whose elements are +1 or -1 according as $\mu_{1}, \mu_{2}, \ldots \mu_{n}$ is an even or odd permutation of $1,2, \ldots, n$.

The most general constant tensor is the algebraic combination of these two tensors. To establish this sesult we observe from eq. (12)

$$
\begin{aligned}
& F_{\mu_{1} k_{2} \ldots \beta_{j-1} \mid(x) p_{j+1} \ldots \beta_{y}}=0
\end{aligned}
$$

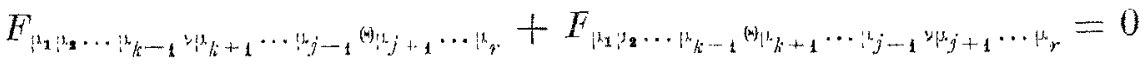

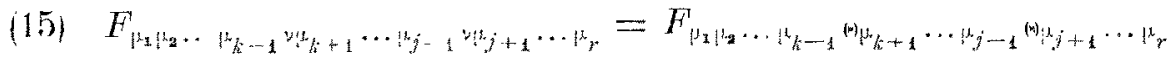

where none of the $\mu$ 's coincide with $\vee$ and $\theta$, which are themselves distinct. From eq. (12) it follows that there cannot be any totally antisymmetric constant tensor of rank less than $n$. Next from eq. (13), it follows that a tensor of rank $n$ whose elements are nonvanishing only when all the indices are distinct is necessarily totally antisymmetric. Finally let us assume that the result is valid for tensors of rank less than $r$. It is easy to show from eqs. $(13)-(15)$ that

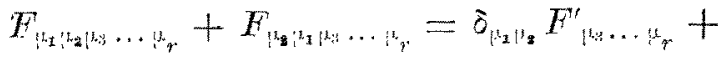

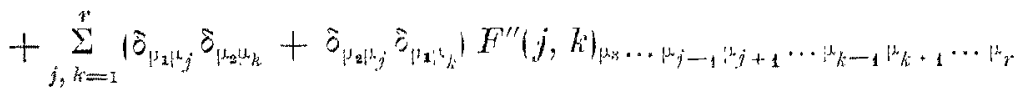


(17)

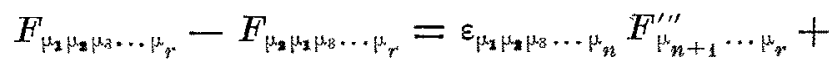

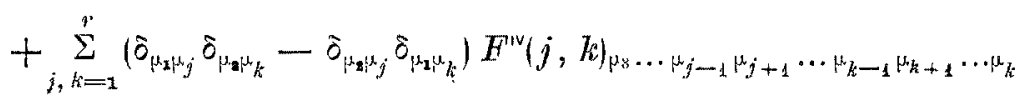

where $F^{\prime \prime \prime}$ is zero if $r<n$ and is a tensor of rank $r-n$ otherwise. Now since all the dashed tensors are of rank less than $r$, our assertion is valid for $r$, if it is valid for tensors of rank less than $r$. This establishes the result for any $r$ since it is true for $r=1$, and $r=2$, which can be verified directly.

\section{3. - Tensors which are functions of vectors.}

Let

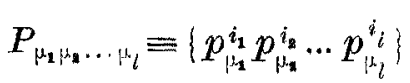

be a tensor of rank $l$, where $p^{2 \prime} s$ are arbitrarily chosen vectors from the set $p^{i}(i=1,2, \ldots m)$ and let the tensor $F_{\mu_{1} \mu_{2} \ldots \mu_{r}}$ be expressed as a sum of terms which are homogeneous in $p_{u}^{i}, s$, except for factors which are functions of invariant scalar products. The eq. (10) points out that each such term should satisfy the differential equation separately. Hence ench term must be separately a tensor of rank $r$, so it must be of the form lexcept for constant coefficients),

$$
P_{\mu_{1} \mu_{2} \ldots \mu_{l}} F_{\mu_{l+1} \ldots \mu_{r}}
$$

where $F_{\mu_{l+1} \ldots p_{r}}$ is a constan tensor of rank $r-l$. Therefore a tensor of rank $r$ whose elemeats are fuctions of vectors is a suitable algebraic combination of tensors $P_{\mu_{2} \mu_{2} \ldots \mu_{7}}, \delta_{\nu \theta}$ and $^{-} \varepsilon_{v_{1} \nu_{2} \ldots v_{n}}$. Hence one can write explicitly the tensors with invariant coefficients. In the following $D$ 's are non-vanishing only when $n=2$ and summations over different vector indices $i$ 's are from $i=1$ to $m$ and the tensor indices $\mu$ 's are from $\mu=1$ to $n$.

$$
\begin{aligned}
& \text { (i) } r=1 ; F_{y}=\sum_{i} A^{i} p_{y}^{i}+\sum_{i} C^{i_{1} i_{2} \ldots i_{n-1} \varepsilon_{y \mu_{2}} \ldots \mu_{n}} p_{\mu_{3}}^{i_{3}} p_{\mu_{3}}^{i_{3}} \ldots p_{\mu_{n}}^{i_{n-1}} \\
& \text { (ii) } \quad r=2 ; F_{\nu \Theta}=\sum_{i} B^{i_{2} i_{2}} p_{\nu}^{i_{2}} p_{\Theta}^{i_{3}}+G \grave{\delta}_{\nu \Theta}+
\end{aligned}
$$

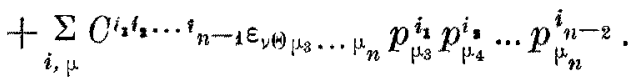

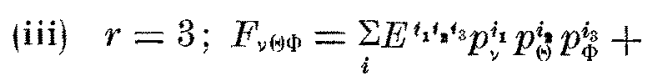

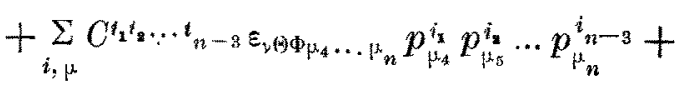

$$
\begin{aligned}
& +\sum_{i}\left\{\left(A_{1}^{i} \delta_{\nu \Theta}+D_{1}^{i} \varepsilon_{\nu \Theta}\right) p_{\Phi}^{i}+\ldots+\ldots\right\} \text {. }
\end{aligned}
$$


(iv)

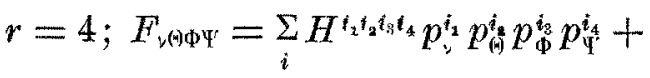

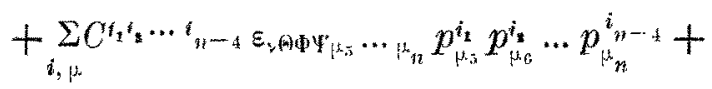

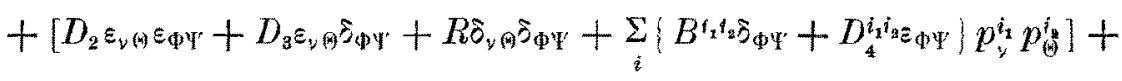

$$
\begin{aligned}
& +\ldots+\ldots+\ldots+\ldots+\ldots
\end{aligned}
$$

The expressions become more cumbersome with increasing $r$. The dots stand for similar terms obtained by permutation of $\nu, \Theta, \Phi, \Psi$. The special case of only one vector, namely the coordinates $x_{\mu k}$, has been discussed by ZiN (") (for $n=3$ and $n=4$ ). In this case

$$
\begin{aligned}
& \text { (i) } r=1 ; f_{\nu}=\alpha x_{\nu}+\Sigma_{\mu} D^{\prime} \varepsilon_{\nu \mu} x_{\mu}
\end{aligned}
$$

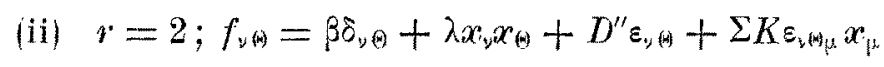

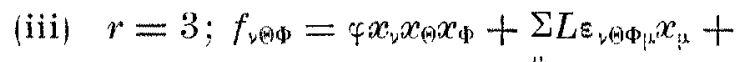

$$
\begin{aligned}
& +\left(A_{1} \delta_{\nu \Theta}+D^{\prime \prime} \varepsilon_{\nu \Theta}\right) x_{\psi}+\ldots+\ldots+\ldots .
\end{aligned}
$$

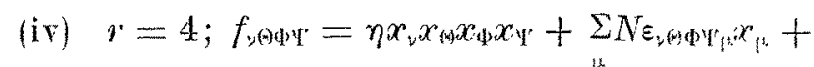

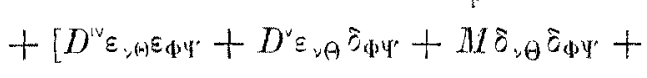

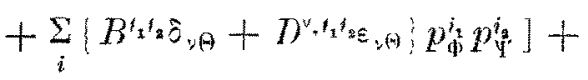

$$
\begin{aligned}
& +\ldots+\ldots+\ldots+\ldots+\ldots
\end{aligned}
$$

In the above $K=0$, when $n \neq 3, L=0$ when $n \neq 4$, and $N=0$ when $n \neq 5$.

I like to thank my friend and colleague Dr, R. Pratap for helpful discussions and the Atomic Energy Establishment, Government of India for their support.

(9) See footnote (1). 